\title{
Anthrovision
}

Vaneasa Online Journal

$2.1 \mid 2014$

Anthropologie et Numérique

\section{Musique improvisée et technologies numériques}

Quelles questions, quels problèmes?

\section{Dario Rudy}

\section{(2) OpenEdition}

Journals

\section{Édition électronique}

URL : http://journals.openedition.org/anthrovision/620

DOI : 10.4000/anthrovision.620

ISSN : 2198-6754

Éditeur

VANEASA - Visual Anthropology Network of European Association of Social Anthropologists

Référence électronique

Dario Rudy, "Musique improvisée et technologies numériques », Anthrovision [En ligne], 2.1 | 2014, mis en ligne le 15 septembre 2014, consulté le 02 mai 2019. URL : http://journals.openedition.org/ anthrovision/620; DOI : 10.4000/anthrovision.620

Ce document a été généré automatiquement le 2 mai 2019.

(c) Anthrovision 


\section{Musique improvisée et technologies numériques}

Quelles questions, quels problèmes?

Dario Rudy

\section{Introduction}

1 En guise d'introduction, il est important de signaler que le point de vue adopté face à l'arsenal technologique essaiera autant que possible de ne se réduire ni à une pensée «déterministe» qui n'attribuerait qu'à la seule technologie les modifications des pratiques musicales - dues à un « système technicien » (Ellul, 1960) ou à une technocratie (Rozsak, 1970) sur lequel les musiciens n'auraient pas de contrôle - ni à une option «instrumentiste », qui verrait dans les outils de simples exécutants de besoins construits socialement. Si ces deux tendances se retrouvent, parfois mélangées ${ }^{1}$, chez les musiciens interrogés, l'option la plus féconde pour notre enquête consiste à concevoir l'outil comme un dispositif modifiant les formes de « prises de décisions » musicales et qui d'une nature épistémologique propre, doit se soumettre à l'épreuve du «direct ", en proposant une forme convenable de jouabilité [playfullness] et d'adaptation à celui qui l'utilise. Il s'agira donc de comprendre de front ce que l'outil « permet » à son usager et son rôle dans le procédé de création de ce dernier. Cette compréhension «à minima » du rôle de l'outil possède un double avantage: celui de proscrire un jugement trop vaste sur «la technologie » et «la musique » ainsi que d'empêcher l'évaluation les pratiques actuelles par des critères esthétiques pré-existants liés à des formes d'actions et d'interactions étrangères à ces outils. Cependant cette proposition se révélerait inopérante si elle nous conduisait uniquement vers une morphologie musicale des objets qui s'interdirait de penser l'évolution des dispositifs musicaux et celles concomitante des pratiques dans un contexte culturel plus ample (les manières de faire de la musique, d'écouter, de danser, la place de l'artiste dans la société, les modifications monde du travail etc.). Le chemin que l'on souhaite suivre implique de moduler le regard critique posé sur l'improvisation dans le monde contemporain aux informations empiriquement récoltées, aux pratiques de 
chacun des musiciens interrogés. Le document proposé ne se fixe pas pour but de découvrir des enjeux « cachés » de la pratique de la musique improvisée, mais d'esquisser, à partir des gestes et des paroles des musiciens et chercheurs interrogés, un cadre interprétatif et des questions réflexives essentiellement heuristiques pour une étude plus poussée à venir.

\section{Le cadre matériel des musiciens}

2 Afin de mieux appréhender les différentes questions que posent les technologies numériques au cadre de l'improvisation, on adoptera ici une logique du concret vers l'abstrait, en nous plaçant tout d'abord à un niveau descriptif qui requiert de détailler chez chacun des protagonistes rencontré le dispositif technique employé.

David Fenech utilise une "loopstation». Cet outil se déclenchant au pied permet d'enregistrer et de garder dans ses mémoires de courtes plages de sons (pas plus de deux minutes) provenant de trois types de sources sonores : en RCA (pour du matériel audio: platine vinyle, cd...) en jack (pour instrument électrique ou électrifié) et en XLR (pour un micro). David Fenech combine les trois types proposés en branchant sur sa pédale à la fois sa guitare, une mini-platine vinyle et un microphone.

Cet outil propose trois types d'action sur le son :

- monter/baisser le volume des sources connectées

- inverser le sens de lecture ou ralentir la lecture

- Enlever/ajouter des boucles de son (jusqu'à 3 phases simultanément)

5 Jean Jacques Birgé a utilisé et continue à utiliser un grand nombre d'instruments électroniques et numériques - il est l'un des premiers à avoir possédé un home-studio en France. Son studio actuel porte la trace de l'histoire récente des technologies musicales : premiers échantillonneurs, premiers synthétiseurs, premiers ordinateurs personnels.

6 Dans ses travaux improvisés les plus récents, avec le trio El Strøm comme dans le duo qu'il forme avec Vincent Ségal, on retrouve quelques invariables dans sa «boite à outils» :

7 Le tenori-on de Yamaha, cadre composé de petites boules lumineuses qui permet à la fois de jouer et de manipuler le son en représentant les sons sous la forme de «trajectoires » que le musicien dévie, renverse. Léger, cet instrument projette sa lumière des deux côtés - à la fois vers les yeux de celui qui le manipule et vers le public.

8 La «mascarade machine » développée spécialement pour J-J Birgé par son ami Antoine Schmitt, est un theremin numérique. Captant par la webcam de l'ordinateur portable les images des mains de J-J Birgé, elle fait correspondre les gestes à un ensemble d'action. Elle permet à la fois de lancer des samples mais aussi de déclencher un flux radiophonique, ou de créer des sons de synthèse à partir des gestes.

9 Ces deux outils sont la plupart du temps utilisés dans un ensemble disparate d'instruments - instruments acoustiques d'Asie du Sud-Est (guimbarde cambodgienne, violon thaillandais) - que Jean Jacques Birgé accumule et auxquels il a dévoué une pièce entière au rangement très précis.

10 Stéphane Rives et Bob Ostertag utilisent tous deux dans leurs projets les plus récents l'ordinateur comme outil d'agencement et de lecture d'échantillons sonores préalablement constitués. Les deux travaillent avec le logiciel Ableton Live. 
11 Chez Stéphane Rives, il s'agit de rejouer des morceaux de sa discographie coupés en fragments de temps égaux et sélectionnés au hasard. La recherche de Bob Ostertag dans son projet le plus récent Book of Hours est plutôt celle d'un agencement d'une musique collective par le biais d'échantillons de chacun des protagonistes (la démarche est assez proche en cela de son projet Say No More).

Benjamin Levy et Raphaël Imbert travaillent ensemble dans le cadre du développement du logiciel Omax à l'Ircam. Benjamin Lévy est celui qui contrôle Omax lors des concerts de la compagnie Nine Spirit et dont le dialogue et la pratique avec Raphaël Imbert permettent d'envisager un certain nombre de sentiers à suivre dans l'utilisation d'Omax.

Jean-Louis Chautemps et Jean Schwarz font ici figure de précurseurs. Le compositeur concret et le saxophoniste ont tous les deux précocement conçu ou participé à des projets mêlant improvisation et technologies d'enregistrement.

La participation de Jean-Louis Chautemps à l'enregistrement et la représentation de JazzEx de Bernard Parmegiani et les projets de Jean Schwarz - notamment avec Michel Portal - permettent mieux de comprendre à quel prix peut s'effectuer

Thor Magnusson est informaticien et musicien, il a élaboré l'environnement Ixi-audio prévu pour une utilisation dans les situations de concert, qui promeut une vision "nonrestrictive " $d u$ rythme et du timbre. Il est également l'auteur d'une thèse sur l'épistémologie des outils musicaux soutenue en 2009 à l'université du Sussex.

\section{Les différentes fonctions de la technologie}

La présentation sommaire effectuée nous permet de montrer l'éventail de fonctions auxquelles répondent les nouvelles technologies de la musique. Si elle sont de nos jours, souvent fondues en un appareil (l'ordinateur), en un logiciel, ou dans les dispositifs des musiciens, en leur sein coexistent deux catégories d'action sur la musique qui touchent à des plans différents.

La première se déroule sur l'axe du temps de la musique. Elle permet la réification du son en un ensemble d'objets " temporels ». Ces objets qui contiennent le temps, permettant la réapparition de mémoire "encapsulées » sont ce que le philosophe Bernard Stiegler nomme des «rétentions tertiaires" (Stiegler, 1994). Cette expression renvoie à la phénoménologie husserlienne (Husserl, 1928) de l'objet temporel (Zeitobjekt). La première « rétention» est celle qui permet de percevoir l'unité d'une séquence de temps (une mélodie plutôt que des notes enchaînées), la seconde permet d'imaginer cette mélodie, de se la représenter, sans l'entendre. La troisième, que théorise Siegler, serait celle qui permet d'entendre la même portion de temps réifiée un nombre potentiellement infini de fois. Entre donc dans cette catégorie tout ce qui touche à l'enregistrement mais aussi à la diffusion du son. L'échantillonnage (sampling), est avant tout la capture temporelle d'un flux déterminé par un objet « temporel ». L'agencement auquel il peut donner lieu (chez Rives ou chez Ostertag par exemple) est donc un agencement de durées, de valeurs temporelles. Cette catégorie doit sa possibilité à l'intégration par la technologie numérique d'une trame temporelle constituée d'unités égales de temps dont nous discuterons les implications dans la suite du document.

La seconde catégorie de technologie se situe sur l'axe du signal sonore. Y appartiennent tous les objets susceptibles de donner naissance à un son ou de le modifier 
qualitativement. On y inclura donc tous les outils ayant trait à l'amplification d'un signal (microphones, amplificateurs), à son traitement qualitatif par des effets (distorsion, delay, écho etc.) ou encore à la synthèse sonore, qui se propose de faire correspondre des données à un son (synthétiseur, claviers midi).

Il s'agit de réussir à comprendre que lorsque ces fonctions se mélangent par le biais des outils qui les réalisent dans les travaux des personnes interrogées, elles se situent toujours dans un certain rapport entre les deux axes. Cette distinction peut paraitre par trop archétypique mais on verra que chacune des deux catégories configure des interactions des musiciens aux instruments et des musiciens aux musiciens de natures assez différentes.

\section{Les formes culturelles des différentes fonctions}

\section{Le signal sonore}

Tout ce qui concerne le travail de modification qualitative se présente le plus souvent sous la forme d'un ajout, d'un complément ou d'une reformulation de formes culturellement appréhendées et historiquement ancrées de créer de la musique. Ainsi l'électrification d'une guitare si elle permet de faire entendre de nouveaux sons, de les traiter d'une manière radicalement nouvelle, se base sur le même primat que la guitare elle même : le déclenchement d'un son par l'action de la main, ou d'un objet sur les cordes ou le corps de l'instrument. Les haut-parleurs qui transmettent la voix de l'orateur dans le stade se présentent avant tout comme des éléments de transmission dans l'espace d'une source déjà donnée. De même, lorsqu'un informaticien traite en directe le timbre d'un instrumentiste qui joue en direct à ses côtés, il se présente lui-même comme un auxiliaire de la chaîne qui relie le jeu instrumental à sa diffusion dans l'espace. Bien sûr, ces dispositifs ne sont pas sans effet sur les manières de jouer, de s'exprimer et comme le fait remarquer Jean-Louis Chautemps, la tendance contemporaine à articuler moins distinctement les mots employés pourrait s'expliquer par la large présence de dispositifs à même de transmettre la voix dans sa plus grande proximité (microphones, téléphones etc.). On pourrait ainsi proposer que les technologies se déroulant sur l'axe du signal ont pour but d'établir un rapport satisfaisant entre une action physique et un transmission sonore. Cette définition volontairement large permet de rassembler différents âges épistémologiques de la technologie musicale : autant l'amplification qui est liée à un âge analogique de la musique dans lequel le signal produit se transmet tel qu'en lui-même d'appareil en appareil que la synthèse qui, ayant pour point de départ des informations épistémologiques sur le son qu'elle doit interpréter - ce que le philosopohe Don Ihde appelle hermeneutical relationship et qu'il oppose à embodied relationship que représente le savoir-faire de l'instrumentiste acoustique (1990) - propose de faire correspondre des paramètres physiques (force de pression d'une touche de piano, durée de cette pression ou encore rotation d'un oscillateur) et une forme d'onde sonore (instrument virtuel).

\section{La contradiction de la synthèse}

C'est par ce canal que se développent des affects - au sens spinoziste du mot qu'utilise Yves Citton de « ce qui s'imprime en moi d'extérieur » - et des affections (« la façon dont s'intègre en moi, dans le côté actif de la sensibilité ») du point de vue du timbre de la 
musique qui définissent notre actualité musicale (la présence de sub-basses dans la musique techno, les sons de synthétiseurs du funk etc). Plus encore, il est évident que c'est par le biais des canaux d'amplification et de traitement du son qu'ont été « découvertes » et « travaillées » certaines formes de micro-réalités sonores (les «bruits » dans la musique concrète ou la gamme de micro-variations dont est capable un saxophone) qui sont ensuite revenues dans la pratique instrumentale "acoustique» comme nous le montre le travail de Stéphane Rives au saxophone.

Mais, cette extension possède une forme de contre-coup: en procédant par réductionnisme pour conceptualiser le son (selon la méthode des sciences naturelles exemplifiée par la classification périodique des éléments de Mendeleiev - référence suggérée par Yves Citton), la technologie musicale de synthèse a donné naissance a des objets qui, pour produire un son ont évacué les propriétés non-nécessaires ou parasites que produisent les instruments acoustiques et qui forment en large part la richesse et par extension la beauté de leur son. La plupart des instruments virtuels que l'on trouve inclus dans les synthétiseurs produisent des spectres sonores assez pauvres et se trouvent inclus et proposés par des objets standardisés dont la vente s'est massifiée depuis les années 1970. Ainsi, incarnée dans des claviers largement diffusés, proposant des sons déjà constitués et peu accommodants, ce que l'on pourrait appelé l'instance d'extension de nos affects et affections musicales a résulté de fait sur un plan quantitatif, dans une forme d'appauvrissement de la diversité des timbres.

\section{La question de l'individuation sonore et l'improvisation}

Ces outils (dont le Yamaha DX7 représente le premier modèle largement diffusé et encore utilisé - on en trouve chez Bernard Lubat, chez Jean Schwarz, chez Jean-Louis Chautemps et chez Jean-Jacques Birgé) ne posent aucun problème à la notion d'improvisation ellemême (l'improvisation étant entendue ici comme un art du temps « réel », se dévoilant en public sous la forme de "performance ») puisqu'ils se plient à l'épreuve du direct et font preuve d'une maniabilité satisfaisante. C'est bien plutôt pour la culture sonore issue du jazz qu'ils font question. Si l'on considère avec George E. Lewis (2005) que le système " afrologique » d'improvisation met en valeur une forme de narration personnelle liée à une relation avec l'outil, qui s'approfondit à l'épreuve du temps, et dont le but ultime est de «trouver son propre son", alors le passage du piano vers le synthétiseur transforme qualitativement ce processus d'individuation sonore en le situant à d'autres niveaux peut être moins au niveau physique de recherche « incarnée » que vers la programmation d'instruments virtuels personnalisés, qui nécessite un autre type de compétence sur le son. À cet égard l'anecdote de Jean-Jacques Birgé sur sa manière de s'approprier les synthétiseurs s'avère parlante :

«J'avais l'habitude, quand je faisais beaucoup de scène, d'enlever le nom de mes instruments, de mettre un scotch. Je le faisais à partir du moment où je pensais que

l'instrument était devenu mien. Que ça ne ressemblait plus aux sons d'usine, que je

l'avais bourré de sons à moi, et donc c'était mon truc et plus Yamaha ou Roland. »

\section{Les technologies du temps}

Les outils dont l'action se situe sur l'axe du temps matérialisé posent en revanche nettement plus problème à la notion même d'improvisation. En substituant une multitude 
de temporalités (celle des sons enregistrés) à l'uniformité du geste instrumental et au processus rectilinéaire d'improvisation, elles contribuent à étendre le cadre temporel dans lequel l'action musicale se déroule et à la remplacer par des formes réticulaires d'organisation. À des interactions manifestes entre musiciens s'ajoutent nombre d'interactions possibles au son enregistré qui peuvent toutes se concevoir comme des rapports à une source sonore originelle. À partir des travaux des musiciens interrogés, on peut différencier les manières dont peuvent se tisser liens signifiants entre le présent de l'improvisation et le son enregistré. Il faut d'abord distinguer deux tendances différentes dans l'utilisation du son enregistré :

1. Un lien d'immédiate reproduction dans lequel le son enregistré sert de support à un son qui s'y superpose. La loopstation utilisée par David Fenech entre dans cette catégorie puisqu'un son de guitare enregistré en direct peut tourner sur lui même et être ornementé de sons d'autres sources. Ce que la technologie numérique a perfectionné dans ce lien est la vitesse d'enregistrement et de rejeu du son, devenue instantanée alors que le magnétophone devait littéralement constituer une boucle avant que le son ne revienne. Ce lien reste donc dans le cadre de la performance sous la forme d'une réinjection sonore d'une source présentée sur scène. Le travail de Raphaël Imbert et Benjamin Lévy avec Omax s'y apparente également.

2. Un lien avec un enregistrement passé que l'on rejoue dans le cadre de la performance : c'est le cas de l'utilisation de disques chez les platinistes, mais aussi de la diffusion de samples par des appareils en tout genre (y compris des jouets - appareils que l'on retrouve chez JeanLouis Chautemps, David Fénech et Jean-Jacques Birgé). Dans ce cas, le rapport à la source sonore peut être investi affectivement de valeurs très différentes (et combinables) dont nous citons quelques unes. Lorsque le musicien agence entre eux des morceaux de temps (de musique) disparates, et procède à une forme de montage :

26 il peut agencer des morceaux provenus d'une ou plusieurs sources spécifiquement constituées pour l'occasion et par là dévoiler sur scène des interactions musicales préexistantes à la performance (comme c'est le cas chez Bob Ostertag lorsqu'il improvise avec des sons de John Zorn qu'il a enregistré et qu'il déclenche depuis son clavier) le répertoire de sample peut servir de toile de fond ou de programme à une performance qui joue avec des sons de lieux, de l'histoire spécifiquement choisis.

Il peut agencer des sources disparates constituées au fil du temps comme les platinistes.

Cette liste non-exhaustive vise à contraster le tableau trop uniforme qui voudrait que le sampling augure le nouvel âge d'une culture entièrement configurable (Sinnreich, 2010) et lise le motif de la «mort de l'auteur dans les musiques électroniques » à un niveau trop évident. Les moyens par lesquels la musique cite d'autres œuvres, dont d'autres «types » de musique peuvent traverser une œuvre sont très nombreux. Ce que modifie les nouvelles technologies semble se situer à un autre niveau : s'il on agrée que la musique n'a pas de référent autre qu'elle même, citer un autre morceau de musique (ou une autre "musique ») passe par des moyens culturellement formés de connotations (telle gamme pour exprimer l'orient, l'incorporation de tels thèmes populaires dans une composition). Ce que le sampling permet, c'est de faire revenir une chose elle-même, en tant que telle et non « codifiée » ou « incorporée » par l'écriture musicale. Autrement dit, la musique peut dénoter un autre morceau de musique existant (ce que Jean-Louis Chautemps appelle un "cadavre », puisqu'il n'est plus confié au musicien de donner vie à la musique mais à l'appareil qui la révèle) $)^{2}$.

29 Il s'agit dès lors de comprendre la pratique du sampling ou de la réinjection comme l'organisation d'un retour qui peut être investi de valeurs affectives, artistiques, 
documentaires très variées. Après avoir montré quel type de répertoire peut faire retour, il convient de s'interroger, dans le cadre de l'improvisation sur les manières dont il peut revenir et des règles décidées par les musiciens quant à leur utilisation des enregistrement.

\section{Les règles}

30 Ce que l'on appelle règle correspond de fait à une forme de limitation qui a pour but de trouver un compromis satisfaisant entre ce qui est crée "sur place» lors de la performance et ce qui a été préparé au préalable. Il s'agit de la recherche d'une contrainte qui délimite le cadre dans lequel les actions vont pouvoir avoir lieu. Par exemple David Fenech se donne pour contrainte de toujours enregistrer le son utilisé comme base en direct, pour que le geste soit exposé et de se limiter dans le temps quand à l'utilisation d'une boucle. Chez Stéphane Rives comme chez Bob Ostertag, il s'agit de ne pas transposer, pas traiter les sons découpés et les utiliser comme tels, pour que se maintienne une forme de hasard, ou de disruption dans le jeu. Dans la plupart des justifications on retrouve ainsi des raisons qui visent à éviter une forme de répétition que propose de lui même, par défaut l'ordinateur. Il s'agit maintenant de se pencher sur les critiques recueillies chez les musiciens, qui concernent en premier lieu, non pas la technologie numérique en générale mais bien l'ordinateur et de voir comment cette « répétition » par défaut est indissociable de l'ordinateur.

\section{L'ordinateur}

31 Dans les paroles des musiciens - mis à part chez Bob Ostertag qui articule en permanence problématiques musicales et explications politiques englobantes - ce n'est pas tant la technologie numérique, qui fait partie de la boîte à outils de tous, que l'ordinateur qui est visé par leurs critiques. Il faut encore préciser que ce n'est pas l'ordinateur comme objet ou comme ensemble de fonctions techniques mais plutôt l'ordinateur comme instrument de musique dont l'intérêt se voit interrogé sur plusieurs plans qu'il convient de séparer et de développer.

\section{L'ordinateur comme interface corporelle}

C'est le point le plus récurrent des critiques. L'ordinateur ne permet pas une négociation physique intéressante pour le spectateur, et tend à transformer le musicien en " poussebouton " (le mot est de J-J Birgé). De fait, le laptop est l'un des seuls instruments qui qui se dresse entre le musicien et ce(ux) qui lui fait (font) face. En cela il s'écarte des formes de représentations précédentes du geste instrumental. L'action des mains, qui se déroule dans un périmètre très réduit est la plupart du temps de toute façon souvent rendue invisible par cet écran qui sépare le musicien du public. Il ne s'agit pas de dire que l'ordinateur ne propose pas de geste, ou encore, que le geste n'a pas d'importance puisque dans la situation d'improvisation (à moins d'un truc) le geste est encore ce qui déclenche l'apparition du son. Mais le peu d'amplitude physique qui accompagne le geste laisse percevoir une question plus profonde quant à la modification qualitative de celui ci dans l'acte de « jouer » de la musique à l'âge épistémologique de la technologie numérique. 
33 Le geste n'est pas chose aisée à définir, et les tentatives de le définir conduisent souvent aux deux positions antagonistes que sont d'un côté ne considérer que l'aspect ultimement physiologique de ce dernier - tel geste du pouce effectué de telle manière - et de l'autre, de devoir considérer le geste comme forme de pensée qui conduit à parler de geste de compositeur, d'interprète etc.

34 Il serait intéressant pour cette étude de réussir à le concevoir comme la pointe d'un triangle qui réunit ensemble une forme de préparation ou de conditionnement du geste et une réalisation physique ponctuelle ${ }^{3}$. Cela ne veut pas dire que tout geste est préparé ni que tout geste prend sens dans une forme de grammaire globale, mais plutôt que le geste prend place dans un ensemble de possibles défini par notre corps, et que ces facultés sont exercées, valorisées, interprétées au quotidien par notre culture et qu'elles configurent en une manière sensible d'appréhender le monde. L'apprentissage de la musique est un exemple de la manière dont les corps sont mobilisés et entraînés à effectuer les gestes avec une certaine force, dans un certain espace à fin d'obtenir quelque chose. Le " discours improvisateur $»^{4}$ - dont les écrits de Derek Bailey ou de Lê Quan Ninh donnent un bon aperçu - lorsqu'il critique le "dressage » auquel donne lieu l'apprentissage classique de la musique, s'oppose non pas à l'idée que le geste se développe, se travaille et permet d'arriver à une fin, mais conteste les enjeux de cette fin (le «beau», la " déférence due au compositeur ») et parie sur une forme d'individuation par le geste qui permette l'expression de court-circuits dans la relation du geste à sa programmation (pouvoir "sortir de soi », " des langages » musicaux, intégrer le cadre et les affordances qu'il propose...).

Si l'on accepte à présent la différence épistémologique - basée sur la phénoménologie de la technique de Don Ihde (Ibid) - par notre interlocuteur Thor Magnusson (2011) entre d'un côté l'instrument acoustique qui propose aux musiciens un savoir avant tout tacite et corporel sur le son, creusé par l'expérience et de l'autre les outils récents - comme le synthétiseur ou l'ordinateur - qui partent d'information épistémiques sur le son avant de le produire, dont on doit interpréter les informations (et c'est littéralement ce que fait Benjamin Lévy par exemple lorsqu'il travaille avec Raphaël Imbert), on peut réussir à mieux saisir cette modification qualitative du geste que donne à percevoir le joueur de laptop.

Le geste prend place dans un ensemble d'informations sur le son qui font appel à une interprétation et à une compétence détachée de l'action de jouer de la musique ellemême. Le geste se donne ainsi la plupart du temps à voir littéralement comme "déclenchement» d'un processus de programmation beaucoup plus long et ne nécessitant le corps qu'à minima (former une banque de son, préparer un sequencer avec un certain nombre de piste, créer des patches sur Max/MSP par exemple). Il intervient donc en dernier ressort d'un processus interprétatif qui fait appel à une autre forme d'interaction avec le son.

Mais, les arguments opposés à la thèse de Magnusson par Green (2011) s'avèrent également intéressants à considérer et nous permettent d'esquisser plus finement le tableau: certes, le geste prend forme sur une trame épistémologique différent mais le dispositif sur lequel il agit doit tout de même répondre à des critères de jouabilité [playfullness] et d'adaptation. Les outils électroniques proposent une représentation graphique ou incarnée du son sur une surface qui répond aux besoins et modulent les travaux des improvisateurs. Autrement dit, ce n'est pas parce que les outils numériques 
font appel à des compétences avant tout interprétatives de l'ordre d'un savoir scientifique sur le son que leur constitution physique est indifférente.

\section{L'ordinateur comme outil musical} termes physiques et de rapidité d'action (connaissance de tous les raccourcis claviers par exemple) mais à cela s'oppose un trait propre aux outils technologiques récents que David Fenech suggère : le très rapide renouvellement de produits disponibles sur le marché et les évolutions également rapides en terme d'actions possible sur le son - qui les rendent indispensables aux musiciens parce que plus commodes ou réunissant en leur sein ce que plusieurs programmes réalisaient séparément - balaye les possibilités d'expression de ces connaissances tacites de l'instrument informatique à quelques exceptions prêts. C'est là tout le sens de l'exemple donné par David Fenech de l'improvisateur Américain Tim Perkis qui utilise le même système depuis 25 ans dont il est à même de maîtriser tous les leviers.

Ainsi, il existe et il peut exister une forme de connaissance « incarnée » des programmes informatiques ${ }^{5}$. Celle-ci réside en une connaissance physique de l'univers de contraintes de l'action que les programmes proposent (un des exemples les plus parlant en serait le type de virtuosité que l'on rencontre chez les "gamers»). Cependant ce type de connaissance est souvent délaissé au profit d'une compétence plus générale qui permet d'identifier les particularités de chacun des programmes et de les combiner les actions sur le son les plus diverses et les plus sophistiquées. Cette manière d'agencer des données épistémiques entre-elles que promeut l'ordinateur en fait pour Jean-Louis Chautemps un outil apollinien, alors que le jazz, ou l'élément «organique» de la musique vivante descendrait de Marsyas, qui ramassant l'Aulos - instrument à anche - qu'Athéna avait inventé puis jeté car il lui déformait le visage, défie Apollon - le joueur de lyre - dans un concours présidé par le roi Midas et par les Muses. Apollon recueille l'admiration collective en chantant accompagné de sa lyre. Après de nombreuses discussions sur les règles du concours et les trois épreuves achevées, les Muses déclarent Apollon vainqueur bien que le roi Midas penche en faveur de Marsyas. Apollon punit le «souffleur» de son outrecuidance - le débat l'avait irrité - en le faisant écorcher au fond d'une grotte. Pour l'avoir soutenu, le roi Midas reçoit une paire d'oreilles d'ânes ${ }^{6}$. La victoire du savoir désincarné de l'ordinateur contre la pratique corporelle du saxophone serait pour JeanLouis Chautemps le signe de notre modernité musicale.

\section{L'agentivité de l'ordinateur}

Ce détour «mythologique » permet de joindre le deuxième point de le plus fréquent de la critique adressée par nos interlocuteurs à l'ordinateur: celle d'un monde «sans contraintes ", "sans fin ». Cela tient au fait qu'il ne soit pas utilisé comme un univers de contraintes physiques définies mais comme un outil d'agencement des divers effet sur le son. Le choix des actions sur le son est potentiellement infini. Si l'on peut lire cela à un niveau politique comme Bob Ostertag qui rapproche cette absence de contrainte au contexte social actuel, dans lequel «nous ne considérons comme réel que ce qui ne nous laisse aucun autre choix $»^{7}$, on peut aussi le lire à un niveau sonore : l'ordinateur n'exerce pas de contrainte (au sens d'effet ou de dégradation) sur le signal sonore qu'il codifie. Le 
son qui sort d'un ordinateur se donne toujours à entendre dans une forme de transparence. Il n'y a pas d'équivalent numérique aux craquements de la poussière sur le diamant d'un disque 33 tours, ou au souffle d'un enregistreur à bandes magnétiques. Or, le plaisir de l'art, comme le rappelle Yves Citton tient en grande partie à ce qu'il « opacifie la transparence supposée du médium qui le reproduit». De fait, comme le montre également l'expérience de Bob Ostertag en tant que juré d'Ars Electronica ${ }^{8}$, il n'est pas exactement d'effet sonore qui soit obtenu par l'ordinateur qui soit immédiatement reconnaissable comme tel, ou qui ne puisse être réalisé par d'autres appareils d'un autre âge technologique. L'effet (ou l'agentivité ) principal de l'ordinateur sur la production musicale, amplement répandu dans tous les genres de musique est « la répétition exacte qu'il est capable de produire » (Ostertag, 1999). Pour Ostertag, cette forme de division du temps en unités égales inscrit l'ordinateur dans la lignée de ce qui constitue selon Lewis Mumford (1976) le véritable point de départ de la révolution technologique : l'invention au Moyen-Âge de l'horloge, et la division du temps en fractions égales. Dans la musique l'introduction d'une forme de "temps » qui contraste avec la manière dont la partition organise une "durée", modulable à ses conditions d'exécution et ouverte à une interprétation offre le terrain d'une investigation philosophique importante. Dans le cadre de la production, elle est incorporée au programmes informatiques et, aussi bien des formes complexes de polyrythmies (comme chez Aphex Twin par exemple) que la répétition la plus basique se basent sur cette donnée.

41 La «réification du temps" opérée par l'ordinateur permet à la fois une grande maniabilité des données temporelles mais influe également sur la perception et la conception des flux temporels. Bob Ostertag parle à propos du travail qu'il a mené avec le logiciel Live comme d'un véritable combat contre la répétition proposée " par défaut " par le programme.

42 Cela n'a pas seulement un effet sur la production musicale mais aussi sur la réception et la manière d'entendre la musique. Ainsi, la répétition parfaite d'un morceau de temps, ou le rythme parfaitement constant d'une grosse caisse synthétique sont devenus la marque ou la trace audible de la présence d'un ordinateur'.

\section{Coda : Omax}

43 Au sein du contexte décrit, le projet Omax semble avoir assimilé les nombreux points critiques et proposer des formes de compromis intéressantes. Le logiciel fait voir l' agentivité propre à l'ordinateur en organisant son action sur le son comme une forme de répétition. Si ce type de travail peut laisser certains sceptiques du point de vue de la fin ou des résultats obtenus, en tant que l'action de l'ordinateur se situe dans un traitement d'une source présente basée sur une forme de répétition, elle est identifiable par le spectateur et ne pose pas de problème au niveau du scanning qui constitue l'une des premières actions du spectateur de musique selon Christopher Smalls. De plus, le programme propose de sortir de cette répétition par défaut en travaillant à la modification du son enregistré en sortie de chaîne, selon une "dialectique d'imitation et de transformation » qui permet de dépasser la forme cyclique de la boucle informatisée.

En outre, l'outil est assez souple pour pouvoir lui faire traiter des types de sons pour lequel il n'est à priori pas prévu, comme les discours de Martin Luther King que fait jouer, 
répéter et déformer Raphaël Imbert à Benjamin Lévy et sur lequel va se superposer un blues joué par le groupe. Il passe ainsi l'épreuve de l'indiscipline aux consignes prévues.

Le point le plus critique à l'heure actuelle semble se situer au niveau du timbre. Si l'ordinateur réplique et modifie ce qui est joué, les terminaux acoustiques qui transmettent le son en bout de chaîne le propagent en modifiant sa résonance et en montrant en creux une différence avec l'instrument acoustique qui n'est peut-être pas suffisamment travaillée, en ce qu'elle montre encore une volonté de transparence de la médiation sur le son effectuée par l'ordinateur (le son serait modifié dans certaines de ses caractéristiques mais pas dans son timbre). Ce constat peut orienter vers une recherche de solutions acoustiques reproduisant plus fidèlement la source enregistré mais elle peut aussi, et c'est là notre préférence, opacifier la médiation de l'ordinateur en lui ajoutant une visibilité et un caractère spectaculaire en mettant en scène les objets transmettant le son originalement capté .

\section{Conclusion}

Ce texte n'aura fait qu'effleurer les vastes questions que peut se poser une anthropologie des modes d'improvisations contemporains et les multiples terrains offerts par les pratiques musicales actuelle. L'enquête réalisée, malgré le nombre peu élevé de protagonistes interrogés nous aura confronté aux questions les plus ardentes du champ. Il semble nécessaire d'en retenir les éléments suivants pour l'élaboration d'une anthropologie de l'improvisation « électronique » contemporaine :

47 l"enquête sur la performance musicale à l'ère digitale doit se moduler à celle-ci et interroger ses conditions de possibilités, les savoirs incorporés, le savoir sur et les interactions au son proposées par les outils utilisés.

Ce qui semble se jouer d'abord dans un type de rapport du corps à l'instrument doit être relié aux procédés d'individuation que permettent les objets techniques (embodied vs hermeneutical).

Elle doit en fin de compte, prendre la mesure d'une forme de rupture qualitative dans les procédés de décisions de l'improvisation "électroniques » et ainsi pouvoir se saisir des des continuités - rapports aux autres, lien à la performance, discours critique - entre l'improvisation telle qu'elle a pu voir le jour dans les années 1960 et ses formes contemporaines et numérisées.

50 Le passage d'une construction incarnée du temps de la musique vers une construction extérieure par une trame sous-jacente et extériorisée est l'une des modification épistémologiques les plus importantes de la production musicale récente, elle implique une forme de rupture avec le jazz et ses catégories esthétiques et dessine de nouveaux espaces de résistances de l'improvisation contemporaine face aux outils et à leur usage dans la production musicale dominante.

51 Cette observation doit pouvoir se relier à des données politiques et sociologiques sur le monde contemporain pour pouvoir servir de levier à une approche des phénomènes sociaux et musicaux actuels notamment par le biais de la notion de rythme (Barthes, 2002 ; Lefèbvre 1992; Michon, 2007). Elle peut également servir de biais intéressant à une enquête sur le monde du travail post-fordiste dans lequel la production s'apparente à une forme de performance. La littérature des sciences de l'organisation (ou management) 
recourt souvent à la métaphore de l'improvisation et du jazz pour désigner l'état d'esprit attendu des salariés (Séité, 2010).

\section{BIBLIOGRAPHIE}

Adorno, T.W., and Levin, T.Y., 1990. The Form of the Phonograph Record. October, Vol. 55.

(Winter, issue), pp. 56-61 [1934].

Chemillier, M., 2009. La transcription musicale à l'ère de l'image animée. À propos des recherches sur l'improvisation et l'ordinateur avec le logiciel OMax (Ircam / Cie Lubat). In Terrain, $\mathrm{n}^{\circ} 53$ Voir la musique.

Ellul, J., 1977. Le système technicien. Paris: Le Cherche-midi.

Green, O., 2011. Agility and Playfulness: Technology and skill in the performance ecosystem, $O$ rganised Sound 16(2)

Idhe, D., 1990. Technology and the Lifeworld: From Garden to Earth. Bloomington :Indiana University Press: 134-144, Cambridge University Press

Ihde, D., 2007. Listening and Voice, Phenomenologies of Sound, Albany, State University of New York Press.

Magnusson, T., 2009. Of Epistemic Tools: musical instruments as cognitive extensions. Organised Sound, 14(2), pp.168-173.

Michon, P., 2007. Les rythmes du politique. Démocratie et capitalisme mondialisé. Paris : Les prairies ordinaires.

Mumford, L., 1976 [1934]. Technique et civilisation. Paris: Le Seuil.

Ostertag B. Why Computer Music Is So Awful. Originally published in published in LMC Texts on Improvised and Experimental Music from Resonance Magazine. Volume 5 Number 1.

Roszak ; T., 1969. The Making of a Counter-Culture. Reflections on the Technocratic Society and its Youthful Opposition. Londres : Faber and Faber.

Sinnrech A., 2010. Mashed up, Music Technology and the Rise of a Configurable Culture. Amherst and Boston : University of Massachussets Press.

Séité, Y., 2010. Critique de l'improvisation. In L'improvisation: ordres et désordres, faits d'art et faits de société. Paris: Université Paris-Diderot (Textuel n60).

Stiegler, B., 1994. La technique et le temps : la faute d'Épiméthée. Paris: Galilée.

Wilson, P. N., 1999. Hear and Now. Hoffheim :Wolke Verlag.

\section{NOTES}

1. Notamment chez Jean-Jacques Birgé qui déclare à la fois : « [un outil] doit m'obéir, faire ce que je lui demande de faire et m'aider à rendre mes rêves faisables » et « je crois que l'outil fait l'art » 
2. Ce à quoi J-J.Birgé oppose le spectacle vivant comme « spectacle fuyant » et donc «spectacle mort » tandis que «[les] disques vivent» .

3. Cette proposition est inspirée par la notion de présent chez Bergson dans La pensée et le mouvant telle qu'elle est réinterprétée par Paolo Virno (1994), pour lequel le présent se situe toujours à la pointe d'un triangle réunissant perception et mémoire.

4. Que l'on peut approcher à bien des égards de ce que Christian Boltanski et Ève Chiapello nomment la critique artiste qui ne vise pas une amélioration sociale par des objectifs identifiés mais critique l'aliénation dont le monde du travail est le lieu. Cf. Boltanski, C., Chiapello, E. 1999. Le nouvel esprit du capitalisme. Paris : Gallimard.

5. Les réponses de Thor Magnusson, qui ne nous étaient pas encore parvenues au moment de rédiger ce paragraphe, confirment notre vue.

6. On trouve un récit de cet épisode dans l'Histoire Universelle de Diodore de Sicile dans le Livre III, chapitre XXX. Une édition en ligne est disponible: http://remacle.org/bloodwolf/historiens/ diodore/livre3.htm

7. On peut aussi, comme je l'ai suggéré à Bob Ostertag qui l'a approuvé rapprocher son constat des analyses psychosociales d'Alain Ehrenberg quant au passage depuis la seconde guerre mondiale d'une société dans lesquels la plupart des maux psychiques sont causés par une surabondance de contraintes sociales à une société dans laquelle la plupart des maux psychiques viennent de la surabondance de possible face auxquels l'individu est confronté.

8. La pièce primée, censée refléter l'état d'avancement de la culture et de la technologie numérique était en fait réalisée en partie avec des synthétiseurs analogiques, ce qui avait soulevé un mini-scandale et poussé Bob Ostertag a écrire son pamphlet intitulé Why Computer Music Sucks. 9. C'est d'ailleurs ainsi que l'on peut reconnaître la présence de Bob Ostertag dans son album Say No More qui consiste en la création d'un groupe par le biais de samples d'enregistrements.

\section{RÉSUMÉS}

L'article suivant se propose de faire tenir ensemble les éléments récoltés pendant la durée d'une enquête réalisée avec une douzaine de musiciens et de chercheurs quant à leurs usages, leurs compréhensions et leur appréciations des outils numériques impliqués dans la production musicale actuelle et plus spécifiquement dans la situation d'improvisation. Il s'agira ici, en procédant par points successifs de questionner les changements épistémologiques de la situation d'improvisation qu'entérinent ces nouveaux outils (ordinateur, pédale de boucle, synthétiseurs ainsi que les diverses interfaces qui les prolongent, les complètent ou les déclenchent), les processus d'individuation qu'ils permettent tout autant que les types de réflexes musicaux qu'ils sollicitent.

Resulting from a fieldwork with improvisers, this article aims at identifying and problematising the specifity of the uses of digital devices in the course of the improvised musical performance. It questions the epistemological changes that occure with the use of digital tools as well as the processes of individuation these tools allow.

A partir de un trabajo de campo con músicos e investigadores, este artículo se propone identificar y problematizar la especificidad de los usos de las plataformas digitales en la creación de música improvisada. El texto reflexiona sobre los cambios epistemológicos que se plantean 
con el uso de las herramientas digitales, sobre los procesos de individuación que éstas herramientas permiten así como sobre el tipo de reflejos musicales que requieren.

\section{INDEX}

Keywords : musical improvisation, digital tools, epistemology of musical instruments, anthropology, Bob Ostertag, Stéphane Rives, Jean-Louis Chautemps, David Fenech, Benjamin Levy, Raphaël Imbert, Jean-Jacques Birgé, Thor Magnusson

Mots-clés : improvisation musicale, outils numériques, épistémologie des instruments de musique, anthropologie, Bob Ostertag, Stéphane Rives, Jean-Louis Chautemps, David Fenech, Benjamin Levy, Raphaël Imbert, Jean-Jacques Birgé, Thor Magnusson

Palabras claves : improvisación musical, herramientas digitales, epistemología de los instrumentos musicales, antropología, Bob Ostertag, Stéphane Rives, Jean-Louis Chautemps, David Fenech, Benjamin Levy, Raphaël Imbert, Jean-Jacques Birgé, Thor Magnusson

\section{AUTEUR}

\section{DARIO RUDY}

EHESS

dariorudy@hotmail.com 